\title{
Adaptación de cofias Co-Cr fabricadas por colado por inducción y fusión selectiva láser en CAD/CAM
}

\author{
Adaptation of Co-Cr copings made by induction casting and selective laser melting in CAD/CAM \\ Sheila Mariela Salvatierra Sevillano ${ }^{1, a ; 2, \mathbf{b}}$, Karolay Elizabeth Rodriguez Carpio 3 ,a; ;,b \\ Diana Esmeralda Castillo Andamayo ${ }^{2, a, b, c ; 4, d, ~ M a r t i ́ n ~ G i l b e r t o ~ Q u i n t a n a ~ d e l ~ S o l a r ~ 2, ~ a, b, d ;,, d ~}$
}

\begin{abstract}
RESUMEN
Existen pocos estudios que comparen la adaptación de cofias de Cobalto-Cromo (Co-Cr) fabricadas por la técnica de cera perdida colada por centrifugación por inducción (CPCI) y fusión selectiva láser (SLM) en diseño y fabricación asistida por computador (CAD/CAM). Objetivo: Evaluar in vitro la adaptación marginal e interna de cofias unitarias de aleación Co-Cr sobre una línea de terminación tipo chamfer, fabricadas con la técnica CPCI y SLM en CAD/CAM. Material y Métodos: Se fabricaron 13 cofias de aleación Co-Cr para cada técnica. Se evaluó la adaptación marginal e interna utilizando la técnica de réplica de silicona. Cada réplica fue seccionada en sentido vestíbulo-palatino y mesio-distal para luego ser analizadas a nivel cervical, axial y oclusal mediante un estéreomicroscopio con aumento de 40X. Resultados: La discrepancia marginal de las cofias CoCr fabricadas por la técnica SLM fue $38.1 \mu \mathrm{m}$ y para la técnica CPCI fue $42,22 \mu \mathrm{m}$ sin diferencias significativas ( $>00,05)$. La discrepancia axial para las cofias fabricadas por técnica CPCI fue de $42,02 \mu \mathrm{m}$ y para la técnica SLM de $60,16 \mu \mathrm{m}$ con diferencia significativa $(\mathrm{p}<0.05)$. La discrepancia oclusal para las cofias confeccionadas por la técnica CPCI fue de $101,94 \mu \mathrm{m}$ y para la técnica SLM fue de $232,13 \mu \mathrm{m}$ con diferencia significativa $(\mathrm{p}<0,05)$. Conclusiones: La adaptación marginal fue similar para las técnicas CPCI y SLM pero sin diferencias estadísticamente significativas. La adaptación interna fue mejor para la técnica CPCI que para la técnica SLM con diferencias estadísticamente significativas.
\end{abstract}

PALABRAS CLAVE: Adaptación, diseño asistido por computador, láseres de estado sólido.

\section{SUMMARY}

There are few studies comparing the fit of cobalt-chromium copings ( $\mathrm{Co}-\mathrm{Cr}$ ) manufactured by induction centrifugal casting technique and selective laser melting (SLM) in CAD/CAM. Objective: The purpose of this study was to evaluate in vitro the marginal and internal fit of $\mathrm{Co}-\mathrm{Cr}$ alloy unit copings on a chamfer termination line manufactured by induction centrifugal casting technique (CPCI) and selective laser melting (SLM) in CAD/CAM. Material and methods: Thirteen Co-Cr unit copings were made for each technique. Marginal and internal fit were evaluated with the replica technique. Each sample was cross sectioned in the vestibular-palatine and mesio-distal direction, then observed in a stereomicroscope at a 40X magnification and measured at the cervical, axial and occlusal areas. Results: The marginal discrepancy of $\mathrm{Co}-\mathrm{Cr}$ copings manufactured by the SLM technique was $38.1 \mu \mathrm{m}$ and for the CPCI technique it was $42.22 \mu \mathrm{m}$ without significant differences $(\mathrm{p}>$ 0.05). The axial discrepancy for the copings manufactured by CPCI technique was $42.02 \mu \mathrm{m}$ and for the SLM technique of $60.16 \mu \mathrm{m}$ with significant difference $(\mathrm{P}<0.05)$. The occlusal discrepancy for the copings made by the CPCI technique was $101.94 \mu \mathrm{m}$ and for the SLM technique was $232.13 \mu \mathrm{m}$ with significant difference $(\mathrm{P}<$ 0.05). Conclusions: The marginal adaptation was similar for CPCI and SLM techniques but without statistically significant differences. The internal adaptation was better for the CPCI technique than for the SLM technique with statistically significant differences.

KEYWORDS: Adaptation, computer-aided design, lasers solid-state.

Facultad de Odontología, Universidad Nacional Federico Villareal. Lima, Perú.

Facultad de Estomatología, Universidad Peruana Cayetano Heredia. Lima, Perú.

Facultad de Odontología, Universidad Católica de Santa María. Arequipa, Perú.

Departamento Académico de Odontología Social, Facultad de Estomatología, Universidad Peruana Cayetano Heredia. Lima, Perú.

Departamento Académico de Clínica Estomatológica, Facultad de Estomatología, Universidad Peruana Cayetano Heredia. Lima, Perú.

Cirujano Dentista; b Especialista en Rehabilitación Oral; ' Magister en Estomatología; d Docente. 


\section{INTRODUCCIÓN}

Existen cuatro criterios principales para el éxito de una restauración como la biocompatibilidad, estética, resistencia a la fractura y la adaptación marginal e interna $(1,2)$. Una pobre adaptación provocaría complicaciones biológicas, mecánicas, periodontales y estéticas. La adaptación marginal se describe como "la exactitud con la que encaja el margen cervical de una restauración indirecta sobre una línea de terminación" (3). Se evalúa mediante la discrepancia marginal que es la distancia entre ambos puntos. Autores indican valores entre 100 a $120 \mu \mathrm{m}$ como clínicamente aceptables para una longevidad clínica (4). La adaptación interna es la exactitud con la que encaja una restauración sobre la preparación dentaria (5). Se evalúa al medir la discrepancia interna que es la distancia perpendicular desde la superficie interna de la restauración hacia la superficie oclusal o axial de la preparación $(6,7)$. Esta distancia debe ser uniforme y suficiente para proporcionar el espacio adecuado para el agente cementante y facilitar la colocación de la restauración sin afectar la retención y resistencia (8). No existe un acuerdo sobre el valor adecuado de discrepancia interna; sin embargo, en la gran mayoria de estudios los valores oscilan entre 50 a $350 \mu \mathrm{m}$ (9-13).

Existen diversas líneas de terminación para coronas metal cerámica como por ejemplo chamfer, hombro biselado y bisel de $135^{\circ}$; sin embargo la mayoría de autores considera que la terminación ideal es chámfer (14-17). El material utilizado antiguamente para coronas metal cerámica fueron las aleaciones nobles. Sin embargo, debido a su alto costo, surgen aleaciones que contienen $\mathrm{Ni}$ (Niquel), $\mathrm{Cr}$ (Cromo) y Be (Berilio) pero su uso no es recomendado por la toxicidad del Be y reacciones alérgicas del Ni $(18,19)$. Debido a ello, las aleaciones Cobalto- Cromo (Co$\mathrm{Cr}$ ) se han venido utilizando como una alternativa con mayor resistencia a la corrosión, mejores propiedades mecánicas y mayor biocompatilidad (19).

Para la fabricación de estructuras metálicas existen técnicas como la técnica de la cera perdida colada por centrifugación convencional (soplete) o inducción (CPCI). En la técnica CPCI, el metal es fundido por inducción en un crisol calentado por electricidad y es impulsado por centrifugación o presión al vacío hacia el molde a través de un conducto llamado bebedero $(20,21)$ lo que permite un mayor control de la temperatura de fusión, disminuye la oxidación y contaminación del metal. Otra técnica es la fusión selectiva laser (SLM) mediante (CAD/CAM). Esta es una técnica aditiva que utiliza escáneres clínicos o de laboratorio para capturar la preparación dentaria y diseñar la estructura por medio de un software en computador y luego mediante la aplicación de un láser de granate de aluminio de itrio dopado con neodimio (Nd-YAG) se fusiona selectivamente un polvo de aleación Co-Cr y se construye capa por capa la estructura metálica en 3D $(22,5)$. Este sistema ofrece ventajas como la obtención de cofias metálicas con propiedades físicas y químicas satisfactorias, disminución en el tiempo de fabricación y costo, reducción de errores humanos, previene distorsiones y defectos inherentes a la técnica de cera perdida para que la adaptación de las estructuras metálicas sea óptima $(11,23,24)$.

$\mathrm{Xu}$ et al., encontraron que las cofias fabricadas por SLM presentaban mejor adaptación marginal en comparación con aquellas obtenidas por CPCI con diferencias significativas (23). Huang et al., concluyeron que la adaptación marginal de las cofias $\mathrm{Co}-\mathrm{Cr}$ por SLM fue similar a las cofias Au-Pt coladas y fue mejor al de las cofias Co-Cr coladas (11). En cuanto a la discrepancia axial no se presentaron diferencias significativas entre los grupos. A nivel oclusal la discrepancia fue mayor en las cofias $\mathrm{Co}-\mathrm{Cr}$ por SLM.

El objetivo de este estudio fue evaluar in vitro la adaptación marginal e interna de cofias metálicas unitarias de aleación $\mathrm{Co}-\mathrm{Cr}$ sobre una línea de terminación tipo chamfer fabricadas por las técnicas CPCI y SLM en CAD/CAM.

\section{MATERIAL Y MÉTODOS}

El presente estudio fue de tipo experimental In vitro. Se diseñó virtualmente un modelo maestro de un premolar superior en el cual se simuló una preparación dentaria con ángulo de convergencia total de $12^{\circ}$, cúspide vestibular de $6 \mathrm{~mm}$ de alto y cúspide palatina de $5 \mathrm{~mm}$ de alto desde la línea de terminación hasta la punta de cúspide, tipo de terminación chamfer de $1 \mathrm{~mm}$; la curvatura de la línea de terminación proximal fue de $0,8 \mathrm{~mm}$ con respecto a vestibular y lingual. Posteriormente se imprimió el diseño en aleación Co-Cr en SLM Mlab Cusing R (Concept 
Láser, Lichtenfels, Germany)(25) (figura 1 (A)). Luego se realizaron 14 impresiones con silicona de adición fluida y pesada al modelo maestro (13 para la técnica CPCI y 1 para la técnica de SLM). Se realizó el vaceado de modelos en yeso tipo IV, 13 modelos de yeso para la técnica de CPCI y 1 modelo de yeso para la SLM en CAD/CAM. En los modelos de yeso (figura 1 (B)) para la técnica de CPCI se delimitó con lápiz rojo la línea de terminación del margen cervical.

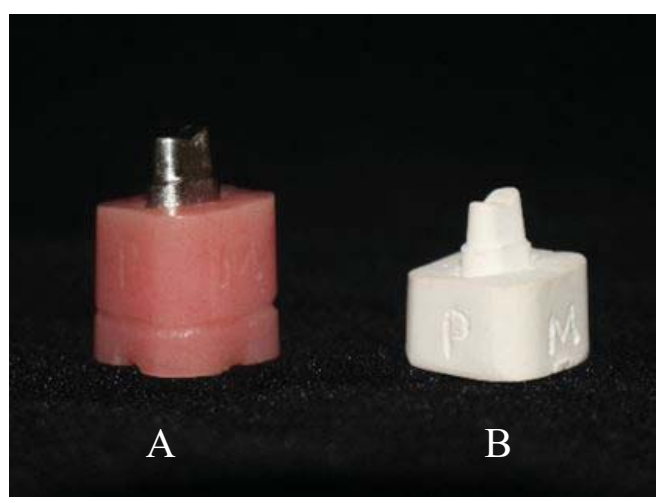

Figura 1. Modelo maestro (A) y modelo de trabajo (B).

Se aplicó un sellador de poros o endurecedor de $0 \mu \mathrm{m}$ llamado die: master dúo (Renfert $\mathrm{GmbH}$, Hilzingen, Germany). Luego se aplicó dos capas de la mezcla de espaciador Pico fit (Renfert $\mathrm{GmbH}$, Hilzingen, Germany) + die: master gold (Renfert $\mathrm{GmbH}$, Hilzingen, Germany) de $20 \mu \mathrm{m}$ a $1 \mathrm{~mm}$ por encima de la línea de terminación y por último se aplicó en todo el muñón una capa de aislante para cera basado en silicona Picosep (Renfert $\mathrm{GmbH}$, Hilzingen, Germany) que presenta una micropelícula de $10 \mu \mathrm{m}$. Se obtuvo finalmente como medidas $10 \mu \mathrm{m}$ en la zona cervical y $50 \mu \mathrm{m}$ en la zona interna (axiales y oclusal) $(5,26)$. Para la confección del patrón de cera, se colocó una capa fina de cera cervical roja StarWax C (Dentaurum GmbH, Ispringen, Germany) en todo el muñón. Posteriormente, se utilizó la técnica de cera por inmersión con el Renfert Hotty (Renfert GmbH, Hilzingen, Alemania) y cera GEODIP (Renfert GmbH, Hilzingen, Alemania). La cofia inicialmente presentó $0.5 \mathrm{~mm}$ de espesor en todas sus caras y se agregó cera para modelar crema StarWax D (Dentaurum $\mathrm{GmbH}$, Ispringen, Germany) solo a nivel oclusal hasta lograr $1 \mathrm{~mm}$ de espesor. Para el sellado de la cofia, se retiró el collarín de cera nivel cervical y luego, utilizando el instrumento PKT Nº 1 se colocó nuevamente cera cervical roja StarWax C (Dentaurum
$\mathrm{GmbH}$, Ispringen, Germany) en el margen cervical y se eliminaron los excesos (27). Se colocó un bebedero de $2 \mathrm{~mm}$ de diámetro en un ángulo de $45^{\circ}$ y una barra difusora de $4 \mathrm{~mm}$ de diámetro al patrón de cera ya formado. Se utilizaron un total de 4 crisoles y se fue colocando 4 patrones de cera por crisol. Se enceraron 16 patrones de cera para que luego de ser coladas, se escogieran 13 cofias según los criterios de selección ya que podía presentarse errores de colado en algunas cofias. Cada crisol fue previamente envaselinado para luego ser revestido con yeso refractario y obtener los cilindros de revestimiento. Cada cilindro se colocó en el horno de calentamiento rápido Ney Vulcan ${ }^{\circledR}$ 3-550 (Dentsplay Sirona Inc, Philadelphia, USA) para la eliminación de cera. Para el colado de la aleación CoCr Cromoron Premium C (DG Dental d.o.o, Gornji Grad, Slovenia) se usó la máquina Fornax ${ }^{\circledR}$ T (BEGO $\mathrm{GmbH}$, Bremen, Alemania) de 3600 VA, la cual es una máquina centrífuga para colado con calentamiento por inducción eléctrica de gran potencia y refrigeración integrada. Se dejó enfriar los cilindros a temperatura ambiente y se realizó el desrevestido del yeso. Luego se verificó el asentamiento de las cofias de forma visual sobre el modelo maestro, las que no ingresaron fueron desgastadas con una fresa de doble punta de carburo tunsgteno hasta conseguir un asentamiento adecuado. Finalmente se procedió a realizar el recorte de los bebederos, el pulido con cauchos y arenado con polvo de óxido de aluminio blanco de $110 \mu \mathrm{m}$ (Protechno, Girona, España) (27). Para la técnica de SLM en CAD/CAM, se escaneó un modelo de yeso con el 3Shape D2000 (3Shape, Copenhagen, Denmark) y se diseñaron las cofias con un grosor de $0.5 \mathrm{~mm}$ en todas sus caras excepto en oclusal donde fue de $1 \mathrm{~mm}$ con el software 3Shape Dental Designer (3Shape A/S, Copenhagen, Denmark). Se usaron las medidas obtenidas del estudio piloto de $60 \mu \mathrm{m}$ en la zona cervical y $70 \mu \mathrm{m}$ en la zona interna (axial y oclusal). Posteriormente, se imprimieron las 13 cofias en 3D con la máquina de SLM MLabCusing (Concept Láser, Lichtenfels, Germany), el cual posee un láser de fibra de Nd-YAG de alta potencia tecnología LaserCUSING ${ }^{\circledR}$ en una atmósfera de Nitrógeno, utilizando el polvo de Co-Cr Remanium ${ }^{\circledR}$ star CL (Dentaurum GmbH, Ispringen, Germany) de 10 a $30 \mu \mathrm{m}$. Se retiraron las cofias y se arenaron con polvo de óxido de aluminio blanco de $110 \mu \mathrm{m}$ (Protechno, Girona, España) a 2 bar de presión (25). Para la evaluación de la adaptación marginal e interna se utilizó la técnica de la réplica de silicona con el 
vinil polieter silicona (VPES) blanco Fit Checker ${ }^{\mathrm{TM}}$ Advance (GC Corporation, Tokyo, Japan), la cual se inyectó en el interior de la cofia y se asentó sobre el modelo maestro a presión constante de $50 \mathrm{~N}$ por 1 minuto con una máquina de ensayos universal Instron CTM-5L (LG Electronics, Seúl, Korea). Luego se retiró la cofia del modelo maestro y se inyectó silicona VPS Elite HD+ Ligth Body Normal Set ${ }^{\circledR}$ (Zhermack Group, Badia Polesine, Italy) (figura 2). Finalmente, cada réplica es cortada en sentido vestíbulo-palatino y luego en sentido mesio-distal (figura 3). Se utilizó un estereomicroscopio Greenough Leica S8 APO (Leica Biosystems GmbH, Wetzlar, Germany) de 40X con el software Leica Application Suite LAS v3.4 (Leica Biosystems GmbH, Wetzlar, Germany) para analizar los fragmentos y medir en micrómetros (figura 4). Se obtuvieron doce medidas por muestra, seis de ellas en sentido vestíbulo - palatino y seis medidas en sentido mesio-distal (25) (figura 5 y figura 6). Se utilizó el programa STATA versión 12 para el análisis univariado y bivariado, donde se evaluaron los supuestos de normalidad con la prueba de Shapiro-Wilk. Se empleó la prueba t de Student para distribución normal y la prueba U Mann-Whitney si no se presentó distribución normal. El presente estudio utilizó un nivel de confianza al 95\% $(\mathrm{p}<0,05)$ y fue aprobado por el Comité Institucional de Ética de la Universidad Peruana Cayetano Heredia.

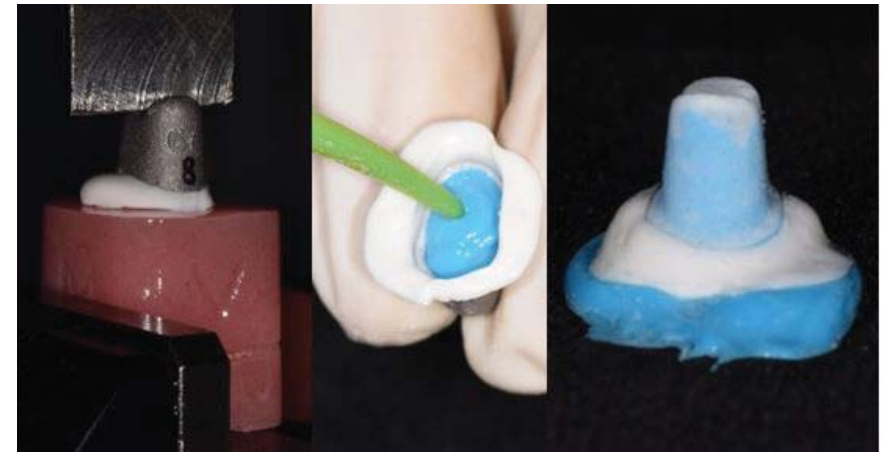

Figura 2. Aplicación del Fit Checker ${ }^{\mathrm{TM}}$ Advance.con presión constante a $50 \mathrm{~N}$ en la máquina Instron y obtención de la réplica de silicona.

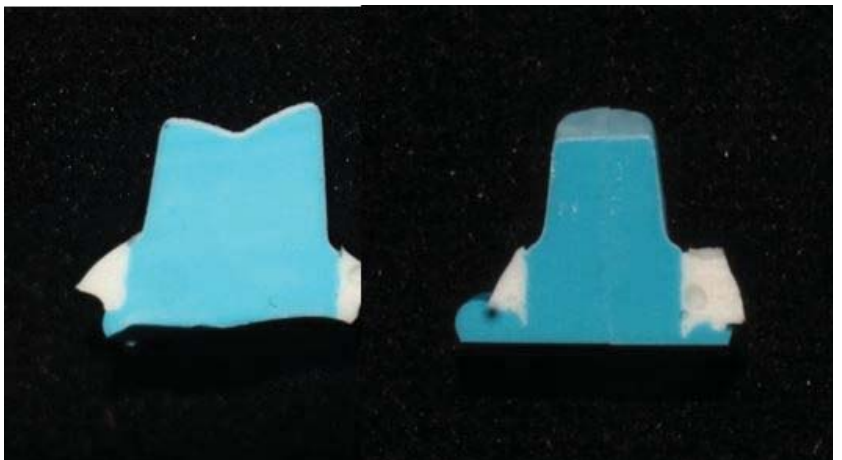

Figura 3. Cortes de la réplica de silicona en sentido vestíbulo palatino y mesiodistal.

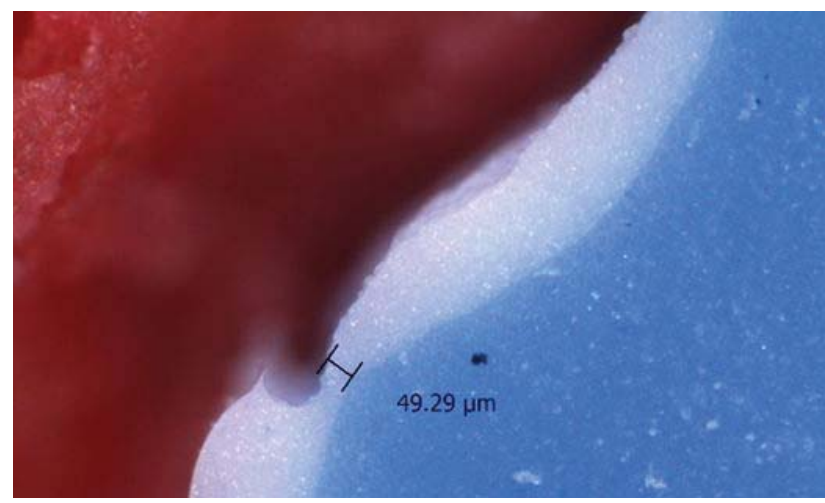

Figura 4. Vista al microscopio y medición en $\mu \mathrm{m}$ de punto marginal. 


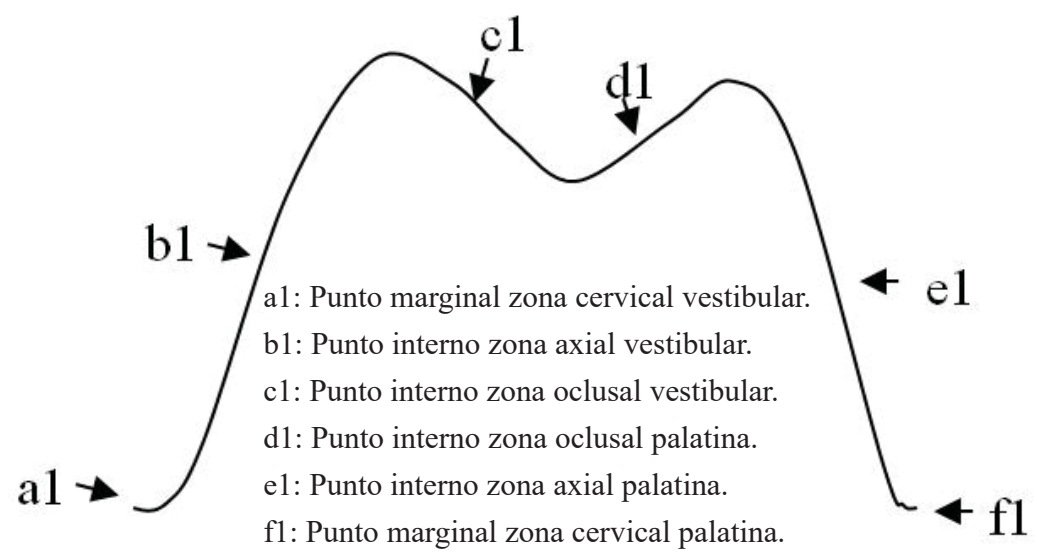

Figura 5. Corte vestíbulo-palatino

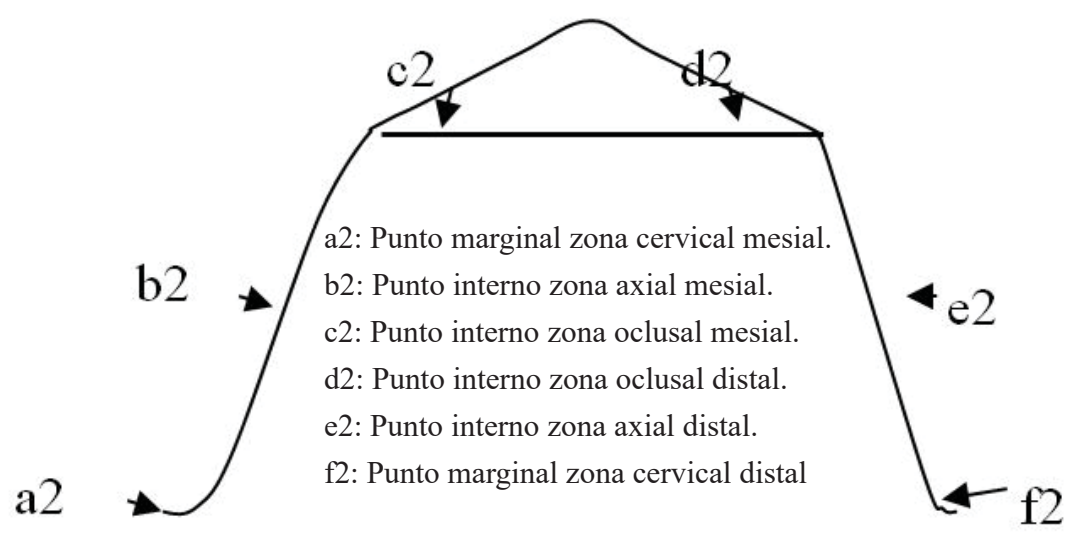

Figura 6. Corte mesio-distal 


\section{RESULTADOS}

En la tabla 1 se observa los resultados descriptivos de la discrepancia marginal de cofias metálicas $\mathrm{Co}-\mathrm{Cr}$ confeccionadas con la técnica CPCI según tipo de corte y punto de medición marginal. El promedio de discrepancia marginal total fue de $42.22 \pm 19.46 \mu \mathrm{m}$.

En la tabla 2 se observa los resultados descriptivos de la discrepancia interna de cofias metálicas fabricadas con técnica CPCI según tipo de corte y punto de medición interno. Se obtuvo que el promedio de discrepancia interna axial fue de $42.03 \pm 12.36 \mu \mathrm{m}$ y de discrepancia oclusal fue de $101.94 \pm 25.61 \mu \mathrm{m}$ en ambos cortes. Además, la discrepancia axial es menor a la discrepancia oclusal, por lo tanto, existe una mejor adaptación a nivel axial que a nivel oclusal. En la tabla 3 se muestran los resultados descriptivos de la discrepancia marginal de cofias metálicas confeccionadas con la técnica de SLM, según el tipo de corte y punto marginal. El promedio de discrepancia marginal total fue de $38.10 \pm 10.26 \mu \mathrm{m}$. En la tabla 4 se puede apreciar los resultados descriptivos de discrepancia interna de cofias metálicas fabricadas con la técnica de SLM, según tipo de corte y punto de medición interno. El promedio de discrepancia interna axial fue $60,16 \pm 14,61 \mu \mathrm{m}$ y de discrepancia oclusal fue de $232,13 \pm 29,83 \mu \mathrm{m}$, se puede observar que los puntos oclusales tienen una dispersión más amplia que los puntos axiales. Además, la discrepancia axial es menor a la discrepancia oclusal, por lo tanto, existe una mejor adaptación a nivel axial que a nivel oclusal. En la tabla 5 se comparó las adaptaciones marginales entre las dos técnicas según tipo de corte y punto de medición marginal y se pudo evidenciar que solo en el corte mesio distal en el punto F2 se obtuvieron valores promedio de $59,82 \mu \mathrm{m}$ para las cofias realizadas con técnica CPCI y de $40,48 \mu \mathrm{m}$ para la técnica de SLM presentando diferencias estadísticamente significativas $(\mathrm{p}=0,003)$. Para los demás puntos de medición no existieron diferencias significativas. La discrepancia marginal total para las cofias fabricadas por la técnica de SLM fue de $38,1 \mu \mathrm{m}$ y para las fabricadas con técnica CPCI fue $42,22 \mu \mathrm{m}$, sin embargo estas diferencias no fueron estadísticamente significativas $(p=0,7998)$. En la tabla 6 se comparó la adaptación interna de las cofias metálicas fabricadas con la técnica CPCI y SLM. Las cofias por técnica CPCI tuvo mejor adaptación interna en comparación a las fabricadas por técnica SML con diferencias significativas $(p<0,0001)$. El promedio de discrepancia axial en las cofias por CPCI fue de $42.02 \mu \mathrm{m}$ y por técnica de SLM fue $60.16 \mu \mathrm{m}$ $(p<0.0001)$. La discrepancia oclusal para las cofias confeccionadas por técnica CPCI fue de $101.94 \mu \mathrm{m}$ y para las de SLM fue de 232,13 $\mu \mathrm{m}(\mathrm{p}<0,0001)$. En general, en ambas técnicas la discrepancia axial fue menor a la discrepancia oclusal.

Tabla 1. Discrepancia marginal de cofias metálicas unitarias de aleación Co-Cr sobre una línea de terminación tipo chamfer fabricada con técnica CPCI según tipo de corte y punto de medición marginal.

\begin{tabular}{ccccccr}
\hline Tipo de corte & $\begin{array}{c}\text { Punto } \\
\text { Marginal }\end{array}$ & Min & Max & Promedio & D.E. & Normalidad \\
\hline \multirow{2}{*}{ Vestíbulo-Palatino } & a1 & 21.00 & 36.14 & 28.30 & 5.02 & 0.51959 \\
& f1 & 23.08 & 47.95 & 30.79 & 6.48 & 0.0335 \\
Mesio-Distal & a2 & 21.00 & 80.86 & 49.96 & 20.52 & 0.30901 \\
& f2 & 34.11 & 85.94 & 59.82 & 19.23 & 0.10764 \\
Discrepancia Marginal Total & & 21.00 & 85.94 & 42.22 & 19.46 & 0.00001 \\
\hline
\end{tabular}

Prueba Shapiro Wilk (normalidad)

Min: mínimo.

Max: máximo.

DE: desviación estándar 
Tabla 2. Discrepancia interna de cofias metálicas unitarias de aleación Co-Cr sobre una línea de terminación tipo chamfer fabricada con técnica CPCI según tipo de corte y punto de medición interno.

\begin{tabular}{|c|c|c|c|c|c|c|c|}
\hline Tipo de corte & Zona interna & $\begin{array}{l}\text { Punto In- } \\
\text { terno }\end{array}$ & Min & Max & Promedio & D.E. & Normalidad \\
\hline \multirow{4}{*}{$\begin{array}{l}\text { Vestíbulo- } \\
\text { Palatino }\end{array}$} & \multirow{2}{*}{ Axial } & b1 & 28.00 & 66.40 & 41.42 & 11.86 & 0.26824 \\
\hline & & e1 & 18.40 & 48.80 & 35.02 & 8.66 & 0.63451 \\
\hline & \multirow{2}{*}{ Oclusal } & $\mathrm{c} 1$ & 54.10 & 128.72 & 85.53 & 19.42 & 0.87994 \\
\hline & & d1 & 64.77 & 140.18 & 105.95 & 23.96 & 0.56585 \\
\hline \multirow{4}{*}{ Mesio-Distal } & \multirow{2}{*}{ Axial } & b2 & 24.80 & 73.60 & 47.89 & 12.33 & 0.99065 \\
\hline & & e2 & 27.21 & 64.00 & 43.77 & 13.70 & 0.06809 \\
\hline & \multirow{2}{*}{ Oclusal } & $\mathrm{c} 2$ & 77.60 & 155.22 & 108.10 & 25.66 & 0.15927 \\
\hline & & $\mathrm{d} 2$ & 58.42 & 151.25 & 108.21 & 28.02 & 0.49504 \\
\hline \multicolumn{2}{|c|}{ Discrepancia Zona Axial } & & 18.40 & 73.60 & 42.03 & 12.36 & 0.33669 \\
\hline \multicolumn{3}{|c|}{ Discrepancia Zona Oclusal } & 54.10 & 155.22 & 101.94 & 25.61 & 0.17068 \\
\hline
\end{tabular}

Prueba Shapiro Wilk (normalidad) Min: mínimo.

Max: máximo.

DE: desviación estándar

Tabla 3. Discrepancia marginal de cofias metálicas unitarias de aleación Co-Cr sobre una línea de terminación tipo chamfer fabricada con técnica de SLM en CAD/CAM, según tipo de corte y punto de medición marginal.

\begin{tabular}{lcccccl}
\hline Tipo de corte & $\begin{array}{c}\text { Punto } \\
\text { Marginal }\end{array}$ & Min & Max & Promedio & D.E. & Normalidad \\
\hline \multirow{2}{*}{ Vestíbulo-Palatino } & $\mathrm{a} 1$ & 23.75 & 36.18 & 31.21 & 4.41 & 0.0593 \\
& $\mathrm{f} 1$ & 22.13 & 77.88 & 36.27 & 13.52 & 0.00038 \\
Mesio-Distal & $\mathrm{a} 2$ & 20.44 & 53.98 & 44.43 & 8.09 & 0.00077 \\
& $\mathrm{f} 2$ & 29.76 & 52.68 & 40.48 & 8.67 & 0.04816 \\
Discrepancia Marginal Total & & 20.44 & 77.88 & 38.10 & 10.26 & 0.00222 \\
\hline
\end{tabular}

Prueba Shapiro Wilk (normalidad) Min: mínimo.

Max: máximo.

DE: desviación estándar 
Tabla 4. Discrepancia interna de cofias metálicas unitarias de aleación Co-Cr sobre una línea de terminación tipo chamfer fabricada con técnica de SLM en CAD/CAM, según tipo de corte y punto de medición interno.

\begin{tabular}{|c|c|c|c|c|c|c|c|}
\hline Tipo de corte & $\begin{array}{c}\text { Zona } \\
\text { interna }\end{array}$ & $\begin{array}{c}\text { Punto } \\
\text { Interno }\end{array}$ & Min & Max & Promedio & D.E. & Normalidad \\
\hline \multirow{4}{*}{ Vestíbulo-Palatino } & \multirow{2}{*}{ Axial } & b1 & 56.80 & 96.81 & 73.85 & 10.88 & 0.64839 \\
\hline & & $\mathrm{e} 1$ & 35.21 & 73.64 & 46.96 & 9.99 & 0.0422 \\
\hline & \multirow{2}{*}{ Oclusal } & $\mathrm{c} 1$ & 186.95 & 287.73 & 224.32 & 32.17 & 0.02069 \\
\hline & & d1 & 196.28 & 318.51 & 243.42 & 32.34 & 0.0291 \\
\hline \multirow{4}{*}{ Mesio-Distal } & \multirow{2}{*}{ Axial } & b2 & 46.40 & 90.40 & 64.50 & 12.69 & 0.44026 \\
\hline & & $\mathrm{e} 2$ & 39.21 & 71.22 & 55.33 & 9.52 & 0.96261 \\
\hline & \multirow{2}{*}{ Oclusal } & $\mathrm{c} 2$ & 191.24 & 300.43 & 234.59 & 28.84 & 0.63385 \\
\hline & & $\mathrm{d} 2$ & 198.41 & 273.42 & 226.18 & 24.89 & 0.08547 \\
\hline \multicolumn{2}{|c|}{ Discrepancia Zona Axial } & & 35.21 & 96.81 & 60.16 & 14.61 & 0.33669 \\
\hline \multicolumn{2}{|c|}{ Discrepancia Zona Oclusal } & & 186.95 & 318.51 & 232.13 & 29.83 & 0.00558 \\
\hline
\end{tabular}

Prueba Shapiro Wilk (normalidad) Min: mínimo.

Max: máximo.

DE: desviación estándar

Tabla N5: Comparación de las adaptaciones marginales de cofias metálicas unitarias de aleación Co-Cr sobre una línea de terminación tipo chamfer fabricadas con técnica CPCI y SLM en CAD/CAM, según tipo de corte y punto de medición marginal.

\begin{tabular}{ccccccc}
\hline Tipo de corte & $\begin{array}{c}\text { Punto } \\
\text { Marginal }\end{array}$ & CPCI & SLM & $\begin{array}{c}\text { Diferencia } \\
\text { de medias }\end{array}$ & valor de p \\
\hline \multirow{2}{*}{ Vestíbulo-Palatino } & $\mathrm{a} 1$ & 28.30 & 31.21 & -2.90 & 0.1297 & $*$ \\
& $\mathrm{f} 1$ & 30.78 & 36.27 & -5.49 & 0.1007 & $* *$ \\
Mesio-Distal & $\mathrm{a} 2$ & 49.96 & 44.43 & 5.53 & 0.555 & $* *$ \\
& $\mathrm{f} 2$ & 59.82 & 40.48 & 19.34 & 0.003 & $*$ \\
Discrepancia Marginal Total & & 42.22 & 38.1 & 4.12 & 0.7998 & $* *$ \\
\hline
\end{tabular}

* Prueba t student $\quad$ ** Prueba U Mann Whitney significancia $\mathrm{p}<0.05$ 
Tabla 6. Comparación de las adaptaciones internas de cofias metálicas unitarias de aleación Co-Cr sobre una línea de terminación tipo chamfer fabricadas con técnica CPCI y SLM en CAD/CAM, según tipo corte y punto de medición interno.

\begin{tabular}{|c|c|c|c|c|c|c|c|}
\hline Tipo de corte & $\begin{array}{c}\text { Zona } \\
\text { interna }\end{array}$ & $\begin{array}{l}\text { Punto } \\
\text { interno }\end{array}$ & CPCI & SLM & $\begin{array}{l}\text { Diferencia } \\
\text { de medias }\end{array}$ & valor de $\mathrm{p}$ & \\
\hline \multirow{4}{*}{ Vestíbulo-Palatino } & \multirow{2}{*}{ Axial } & b1 & 41.42 & 73.85 & -32.43 & $<0.0001$ & $*$ \\
\hline & & e1 & 35.02 & 46.96 & -11.93 & 0.0041 & $* *$ \\
\hline & \multirow{2}{*}{ Oclusal } & $\mathrm{c} 1$ & 85.53 & 224.32 & -138.79 & $<0.0001$ & $* *$ \\
\hline & & $\mathrm{d} 1$ & 105.95 & 243.42 & -137.47 & $<0.0001$ & $* *$ \\
\hline \multirow{4}{*}{ Mesio-Distal } & \multirow{2}{*}{ Axial } & b2 & 47.89 & 64.50 & -16.61 & 0.0025 & $*$ \\
\hline & & e2 & 43.77 & 55.33 & -11.56 & 0.0196 & $*$ \\
\hline & \multirow{2}{*}{ Oclusal } & $\mathrm{c} 2$ & 108.10 & 234.59 & -126.49 & $<0.0001$ & $*$ \\
\hline & & $\mathrm{d} 2$ & 108.21 & 226.18 & -117.98 & $<0.0001$ & $*$ \\
\hline Discrepancia Zona Axial & & & 42.02 & 60.16 & -18.14 & $<0.0001$ & $*$ \\
\hline Discrepancia Zona Oclusal & & & 101.94 & 232.13 & -130.18 & $<0.0001$ & $* *$ \\
\hline
\end{tabular}

* Prueba t student

** Prueba U Mann Whitney significancia $\mathrm{p}<0.05$

\section{DISCUSIÓN}

Los resultados mostraron que la adaptación marginal de las cofias confeccionadas por la técnica de SLM $(38,1 \mu \mathrm{m})$ fueron similares a las cofias fabricadas por técnica CPCI $(42.22 \mu \mathrm{m})$ sin presentar diferencias significativas. Los valores se situaron por debajo de $120 \mu \mathrm{m}$ considerado el límite clínicamente aceptable (4). En cuanto a la adaptación interna, las cofias confeccionadas por la técnica CPCI tuvieron mejor adaptación interna con una discrepancia axial de $42,02 \mu \mathrm{m}$ y oclusal de $101,94 \mu \mathrm{m}$ a diferencia de las cofias por técnica de SLM que tuvieron valores de discrepancia axial de $60,16 \mu \mathrm{m}$ y oclusal de 232,13 $\mu \mathrm{m}$ presentando diferencias significativas. Además, en ambas técnicas los valores de discrepancia fueron mayores a nivel oclusal que a nivel axial. Sin embargo todos se ubicaron en el rango encontrado por diversos autores que van desde 50 a 350 (8,11-13). En general, los resultados no fueron concluyentes para determinar que una técnica es mejor que la otra.

En relación a otros estudios $\mathrm{Xu}$ et al., encontraron que las cofias de Co-Cr fabricadas por técnica SLM presentaban menor discrepancia marginal de 102.86 $\mu \mathrm{m}$ en comparación a la técnica de CPCI de $170.19 \mu \mathrm{m}$ presentado una diferencia significativa (23). Huang et al., concluyeron que las cofias de $\mathrm{Co}-\mathrm{Cr}$ fabricadas por tecnica SLM presentaban mejor adaptación marginal $(75.6 \mu \mathrm{m})$ que las de técnica CPCI (91 $\mu \mathrm{m})$ con diferencia significativa y en cuanto a la discrepancia interna a nivel axial no hubo diferencia, pero a nivel oclusal fue mayor en las cofias de $\mathrm{Co}-\mathrm{Cr}$ fabricadas por técnica SLM (309.8 $\mu \mathrm{m})(11)$.

Como se puede observar con los datos de otros estudios y los encontrados en esta investigación, entre las dos técnicas existen diferentes valores de discrepancias. Si bien las cofias metálicas fabricadas por técnica SLM tuvieron una mejor adaptación marginal que las confeccionadas por técnica CPCI, la diferencia no fue significativa y los valores encontrados estuvieron dentro de los límites clínicamente aceptables. La técnica CPCI permite un mayor control de la temperatura de fusión, disminuye la oxidación y contaminación del metal comparado con la técnica de colado convencional por lo que se puede obtener restauraciones metálicas con microestructuras de grano fino las cuales se caracterizan principalmente por sus mejores propiedades mecánicas y resistencia a la corrosión $(21,22)$. Requena evaluó la adaptación de cofias metálicas Co-Cr confeccionadas mediante dos técnicas de cera perdida colada por centrifugación: convencional y CPCI donde no encontró diferencias significativas (27). En la técnica CPCI, la discrepancia marginal fue de $77.27 \mu \mathrm{m}$, la discrepancia axial de $47,42 \mu \mathrm{m}$ y discrepancia oclusal de $159,05 \mu \mathrm{m}$. Xu et al., mencionan que la adaptación marginal puede 
verse comprometida por factores propios de la técnica de colado como la contracción de la polimerización de la silicona de adición que fue de $0,05 \%$, grado de expansión tanto del yeso tipo IV $0.08 \%$ como del yeso refractario que fue de $3,10 \%$ y la deformación del patrón de cera; mientras que en el proceso de fabricación automática de la técnica SLM estos errores puede prevenirse y crear restauraciones más precisas para uso clínico(23). Otro factor que pudo haber influido es que en este estudio se utilizó un modelo maestro realizado mediante un diseño digital 3D e impreso en SLM con factores controlados de paralelismo de paredes y ángulo de convergencia total de $12^{\circ}$, mientras que otros estudios fueron elaborados por un proceso manual de tallado de un troquel de acero inoxidable o sobre dientes naturales con ángulos de convergencia de $5^{\circ}$ como los estudios de Huang et al., y Xu et al., $(11,23)$. Chang et al., indicaron que ángulos de convergencia menores simplemente pueden no adaptarse y dar lugar a una mayor variación en la discrepancia marginal (28).

En cuanto a la adaptación interna, las cofias confeccionadas por la técnica CPCI tuvieron mejor adaptación interna al compararlas con la técnica de SLM con diferencias significativas. Se cree que pudo deberse a los diferentes espacios para el cemento que se le dio a los grupos, siendo para las cofias de técnica CPCI a nivel marginal de $10 \mu \mathrm{m}$ e interno de $50 \mu \mathrm{m}$ y para la técnica de SLM $60 \mu \mathrm{m}$ marginal y $70 \mu \mathrm{m}$ interno. Asi mismo, Park et al., mencionan que el aumento de la discrepancia interna puede deberse a errores propios de la fabricación automatizada como la entrada de información y la precisión de su procesamiento (29).

Además, los valores de discrepancia oclusal fueron mayores en ambas técnicas sobre todo en las cofias de SLM. Este comportamiento se puede observar en otros estudios como los de Huang et al., Flores y Requena $(5,11,27)$. Huang et al., mencionan que la mayor discrepancia oclusal de la técnica SLM comparadas con las otras técnicas de su estudio pudo deberse a que las partículas de polvo pudieron quedar en la parte interna de la cofia interfiriendo en un buen asentamiento sobre el modelo (11). Kamegawa et al., indicaron que estas diferencias pueden deberse a los escáneres usados en CAD/CAM ya que los modelos de yeso tiene una forma compleja con diversidad de ángulos y se requiere un escáner óptico que sea capaz de hacer varias lecturas en diferentes direcciones para minimizar la mayor cantidad de áreas ciegas por lo que no proporciona una precisión adecuada para fosas y fisuras (30).

Para la evaluación de la adaptación de las cofias se utilizó la técnica de réplica de silicona ya que por su precisión y confiabilidad se ha utilizado en varios estudios $(5,11,23,25-27)$. Se escogió esta técnica por ser un método simple, no invasivo con respecto a las muestras y al modelo maestro. Se realizaron un total de 12 mediciones por cofia como en otros estudios $(5,28)$, otros autores como Groten et al., indican que para evaluar la adaptación de una restauración unitaria se debe medir las discrepancias como mínimo en 50 puntos (31).

Los resultados presentan relevancia clínica, ya que el odontólogo podrá elegir que técnica de fabricación utilizar dado que ambas presentan valores dentro del rango clínico aceptable. La elección y el éxito dependerán de factores como tiempo, costo, comunicación con el técnico dental y una adecuada técnica de fabricación.

Se recomienda realizar estudios clínicos a largo plazo que evalúen la adaptación de cofias de diferentes materiales y sistemas de fabricación, así como el éxito después de la aplicación de la porcelana. Una limitación del estudio fue no haber comparado con otras técnicas de CAD/CAM y no hubo conflicto de interés.

\section{CONCLUSIONES}

Al comparar la adaptación marginal no hubo una diferencia estadísticamente significativa entre ambas técnicas, a pesar que los valores de discrepancia marginal de las cofias de SLM fueron menores que en la técnica CPCI. Al comparar la adaptación interna entre ambos grupos si existió una diferencia estadísticamente significativa tanto a nivel axial como oclusal, siendo los valores de discrepancia interna menores en la técnica CPCI que en la técnica SLM.

\section{Correspondencia}

Sheila Mariela Salvatierra Sevillano

Correo electrónico: sheila.salvatierra.s@upch.pe 


\section{REFERENCIAS BIBLIOGRÁFICAS}

1. Ortega R, Gonzalo E, Gomez-Polo M, Lopez-Suarez C, Suarez MJ. SEM evaluation of the precision of fit of CAD/CAM zirconia and metal-ceramic posterior crowns. Dent Mater J. 2017;36 (4):387-93.

2. Berrendero S, Salido MP, Valverde A, Ferreiroa A, Pradíes G. Influence of conventional and digital intraoral impressions on the fit of CAD/CAMfabricated all-ceramic crowns. Clin Oral Investig. 2016;20 (9):2403-2410.

3. Romeo RM. Estudio comparativo de ajustes en prótesis fija cerámica entre sistemas CAD-CAM e inyectado. Tesis Doctoral.Madrid: Universidad Complutense de Madrid; 2010.

4. Sakornwimon N, Leevailoj C. Clinical marginal fit of zirconia crowns and patients' preferences for impression techniques using intraoral digital scanner versus polyvinyl siloxane material. J Prosthet Dent. 2017;118(3):386-91.

5. Flores Y. Evaluación in vitro de las adaptaciones marginal e interna de cofias metálicas de aleación cobalto cromo (Co-Cr) sobre una línea de terminación tipo chamfer, confeccionadas con dos técnicas: cera pérdida colada por centrifugación convencional y el sistema fusión selectiva por láser asistido por computador (CAD/CAM). Tesis para obtener el título profesional de especialista en Rehabilitación Oral. Lima: Universidad Peruana Cayetano Heredia.; 2017.

6. Holmes JR, Bayne SC, Holland GA, Sulik WD. Considerations in measurement of marginal fit. J Prosthet Dent. 1989;62(4):405-8.

7. Zeltner M, Sailer I, Mühlemann S, Özcan M, Hämmerle $\mathrm{CH}$, Benic GI. Randomized controlled within-subject evaluation of digital and conventional workflows for the fabrication of lithium disilicate single crowns. Part III: marginal and internal fit. J Prosthet Dent. 2017;117(3):354-62.

8. Vojdani M, Torabi K, Farjood E, Khaledi A. Comparison the marginal and internal fit of metal copings cast from wax patterns fabricated by $\mathrm{CAD} /$ CAM and conventional wax up techniques. J Dent (Shiraz). 2013;14(3):118-29.

9. Chochlidakis KM, Papaspyridakos P, Geminiani A, Chen CJ, Feng J, Ercoli C. Digital versus conventional impressions for fixed prosthodontics: A systematic review and meta-analysis. J Prosthet Dent. 2016;116(2):184-90.

10. Eames WB, O’Neal SJ, Monteiro J, Miller C, Roan JD, Cohen KS. Techniques to improve the seating of castings. J Am Dent Assoc. 1978; 96: 432-7.

11. Huang Z, Zhang L, Zhu J, Zhang X. Clinical marginal and internal fit of metal ceramic crowns fabricated with a selective laser melting technology. J Prosthet Dent. 2015; 113(6): 623-7.
12. Örtorp A, Jönsson D, Mouhsen A, Vult-von-Steyern P. The fit of cobalt-chromium three-unit fixed dental prostheses fabricated with four different techniques: a comparative in vitro study. Dent Mater. 2011;27(4):356-63.

13. Nesse H, Ulstein DM, Vaage MM, Øilo M. Internal and marginal fit of cobalt-chromium fixed dental prostheses fabricated with 3 different techniques. J Prosthet Dent. 2015;114(5):686-92.

14. Shillimburg H. Fundamentos esenciales en prótesis fija. 3a ed. Barcelona: Quintessence; 2006.

15. Pegoraro LF. Prótesis Fija. Sao Paulo: Artes Médicas; 2001.

16. Chatterjee U. Margin designs for esthetic restoration: An overview. J Adv Oral Research. 2012; 3(1): 7-12.

17. Bottino MA, Valandro LF, Buso L, Ozcan M. The influence of cervical finish line, internal relief, and cement type on the cervical adaptation of metal crowns. Quintessence Int. 2007; 38(7):425- 32.

18. Roberts HW, Berzins DW, Moore BK, Charlton DG. Metal-ceramic alloys in dentistry: a review. J Prosthodont. 2009;18(2):188-94.

19. Kocaağaoğlu H, Kılınç Hİ, Albayrak H, Kara M. In vitro evaluation of marginal, axial, and occlusal discrepancies in metal ceramic restorations produced with new technologies. . J Prosthet Dent. 2016;116(3):368-74.

20. Abad J, Castro O, Mayken S, Torres E. Comparación de la adaptación marginal de cofias elaboradas en cuatro tipos de máquinas de colado. Rev Odontos. 2014;43:6-15.

21. Gómez-Cogolludo P, Castillo-Oyagüe R, Lynch CD, Suárez-García MJ. Effect of electric arc, gas oxygen torch and induction melting techniques on the marginal accuracy of cast base-metal and noble metalceramic crowns. J Dent. 2013;41(9):826-31.

22. Koutsoukis T, Zinelis S, Eliades G, Al-Wazzan K, Rifaiy MA, Al Jabbari YS. Selective laser melting technique of Co-Cr dental alloys: A review of structure and properties and comparative analysis with other available techniques. J Prosthodont. 2015; 24(4):30312.

23. Xu D, Xiang N, Wei B. The marginal fit of selective laser melting-fabricated metal crowns: an in vitro study. J Prosthet Dent. 2014;112(6):1437-40.

24. Kaleli N, Saraç D. Influence of porcelain firing and cementation on the marginal adaptation of metalceramic restorations prepared by different methods. J Prosthet Dent. 2017;117(5):656-61.

25. Gómez J. Evaluación in vitro de la adaptación de cofias metálicas fabricadas con la técnica fusión selectiva por láser en función del ángulo de convergencia total y el espacio para el cemento. Tesis para obtener el título profesional de Magister en Rehabilitación Oral. Lima: Universidad Peruana Cayetano Heredia.; 2018. 
26. Zelada C. Comparación in vitro de las adaptaciones marginal e interna en cofias metálicas unitarias de aleación cobalto cromo $(\mathrm{Co}-\mathrm{Cr})$ realizadas sobre dos líneas de terminación: tipo chámfer y tipo bisel, fabricadas con las técnicas de la cera perdida colado por centrifugado convencional y de fusión selectiva por láser, diseño asistido por computadora/ manufactura asistida por computadora (CAD/CAM). Tesis para obtener el título profesional de especialista en Rehabilitación Oral. Lima: Universidad Peruana Cayetano Heredia.; 2017.

27. Requena S. Evaluación in vitro de la adaptación marginal e interna de cofias metálicas de aleación cobalto cromo (Co-Cr) sobre una línea de terminación tipo chamfer fabricadas con dos técnicas de cera perdida coladas por centrifugación: convencional y por inducción. Tesis para obtener el título profesional de especialista en Rehabilitación Oral. Lima: Universidad Peruana Cayetano Heredia.; 2018.
28. Chan DC, Wilson AH Jr, Barbe P, Cronin RJ Jr, Chung $\mathrm{C}$, Chung K. Effect of preparation convergence on retention and seating discrepancy of complete veneer crowns. J Oral Rehabil. 2005;32(1):58-64.

29. Park JK, Lee WS, Kim HY, Kim WC, Kim JH. Accuracy evaluation of metal copings fabricated by computer-aided milling and direct metal laser sintering systems. Adv Prosthodont. 2015;7(2):122-8.

30. Kamegawa M, Nakamura M, Tsutsumi S. 3D morphological measurements of dental casts with occlusal relationship using microfocus x-ray. CT Dent mater J. 2008;27(4):549-54.

31. Groten M, Axmann D, Pröbster L, Weber $H$. Determination of the minimum number of marginal gap measurements required for practical in-vitro testing. J Prosthet Dent. 2000 ;83(1):40-9.

Recibido: 15-11-19

Aceptado: 20-04-20 